\title{
Gravitational signals emitted by a point mass orbiting a neutron star: effects of stellar structure.
}

\author{
J.A. Pons ${ }^{1}$, E. Berti ${ }^{2}$, L. Gualtieri ${ }^{1}$, G. Miniutti ${ }^{1}$ and V. Ferrari ${ }^{1}$ \\ ${ }^{1}$ Dipartimento di Fisica "G.Marconi", Università di Roma "La Sapienza" \\ and Sezione INFN ROMA1, piazzale Aldo Moro 2, I-00185 Roma, Italy \\ 2 Department of Physics, Aristotle University of Thessaloniki, Thessaloniki 54006, Greece
}

(October 25, 2018)

\begin{abstract}
The effects that the structure of a neutron star would have on the gravitational emission of a binary system are studied in a perturbative regime, and in the frequency domain. Assuming that a neutron star is perturbed by a point mass moving on a close, circular orbit, we solve the equations of stellar perturbations in general relativity to evaluate the energy lost by the system in gravitational waves. We compare the energy output obtained for different stellar models with that found by assuming that the perturbed object is a black hole with the same mass, and we discuss the role played by the excitation of the stellar modes. Our results indicate that the stellar structure begins to affect the emitted power when the orbital velocity is $v \approx 0.2 c\left(\nu_{G W} \gtrsim 185 \mathrm{~Hz}\right.$ for a binary system composed of two $1.4 M_{\odot}$ neutron stars). We show that the differences between different stellar models and a black hole are due mainly to the excitation of the quasinormal modes of the star. Finally, we discuss to what extent and up to which distance the perturbative approach can be used to describe the interaction of a star and a pointlike massive body.
\end{abstract}

PACS numbers: 04.30.-w, 04.40.Dg 


\section{INTRODUCTION}

In a recent paper [1] (to be referred to hereafter as Paper I) we have studied the gravitational emission of a binary system by using the following perturbative approach: one of the two stars is assumed to be an extended body, whose equilibrium structure is described by an exact solution of the relativistic equations of hydrostatic equilibrium; the second star is a pointlike mass which induces a perturbation on the gravitational field and on the thermodynamical structure of the extended companion. We modeled the extended star using a polytropic equation of state, we chose the parameters so that the radius and the mass were those of a plausible neutron star (NS), and we solved the equations of stellar perturbations to compute the power emitted in gravitational waves when the point mass moves on orbits of arbitrary eccentricity.

In this paper we use the same perturbative approach to compute the power radiated in gravitational waves by different stellar models. The purpose of this investigation is to answer a number of questions that arise in studying the signals emitted by NS-NS binary systems during the last phases before coalescence. The first is about the role played by the internal structure of the star: how does the gravitational emission depend on the mass and radius of the star (and consequently on the equation of state (EOS) of dense matter)? In particular, what is the difference if the perturbed object is a black hole $(\mathrm{BH})$ ? The second is related to the possibility of exciting the quasi normal modes of the star. Are the differences between stars and a black hole (if any) due exclusively to resonant modes or to other orbital effects? The third question concerns the domain of applicability of the perturbative approach: to what extent are the results of this approach applicable to describe the evolution of a true binary system, composed of two neutron stars of comparable mass? Are we still in a perturbative regime when the two neutron stars are, say, 3 or 4 stellar radii apart?

The plan of the paper is the following. In Sec. II we shortly review the procedure we use to find the solution of the equations of stellar perturbations inside and outside the perturbed star, along the lines of Paper I. Since we are mainly interested in the last phases of the orbital evolution, we shall consider the orbit of the point mass as already circularized [2]. In Sec. III we discuss in detail the results of the integration of the perturbed equations for the different stellar models and for a black hole, both excited by the same process. In

Sec. IV we examine the domain of applicability of the perturbative approach applied to binary systems, and in Sec. $\mathrm{V}$ we draw the conclusions.

\section{THE PERTURBED EQUATIONS}

In order to compute the radiation emitted by a a neutron star perturbed by a massive point particle, we need to integrate the equations of stellar perturbations inside and outside the star, and this can be accomplished by using different gauges and formalisms. Inside the star we choose the Chandrasekhar-Ferrari gauge [3], which allows to decouple the equations for the gravitational perturbations from those describing the perturbations of the fluid. After expanding the perturbed metric tensor in tensorial spherical harmonics, choosing 
this gauge the radial part of the polar metric perturbations is described by four functions, $\left[N_{l m}(\omega, r), L_{l m}(\omega, r), V_{l m}(\omega, r), T_{l m}(\omega, r)\right]$, whereas only two functions, $\left[h_{l m}^{0}(\omega, r), h_{l m}^{1}(\omega, r)\right]$, are needed to describe the axial part. As in Paper I, we integrate the equations of stellar perturbations in the frequency domain (cf. Paper I, Eqs. (2.2) and (2.4)) up to the surface of the star, where we construct the Zerilli and the Regge-Wheeler functions, $Z_{l m}^{\text {pol }}(\omega, r)$ and $Z_{l m}^{a x}(\omega, r)$ [4], [5]

$$
\begin{aligned}
& Z_{l m}^{p o l}(\omega, r)=\frac{r}{n r+3 M}\left[3 M V_{l m}(\omega, r)-r L_{l m}(\omega, r)\right], \\
& Z_{l m}^{a x}(\omega, r)=-\frac{e^{2 \nu}}{r} h_{l m}^{1}(\omega, r),
\end{aligned}
$$

and their first derivatives with respect to $r$. The integration of the equations of stellar perturbations in the interior (together with the TOV equation) is done using an adaptive step Runge-Kutta method. The relative tolerance of the integrator is set to $10^{-8}$. We use as integration variable the logarithm of the pressure, which allows us to approach the stellar surface gradually, and to determine the radius with the required accuracy.

The two functions in (2.1) are needed to compute the radial part, $\Psi_{l m}(\omega, r)$, of the perturbation of the Weyl scalar $\delta \Psi_{4}$, which is defined as

$$
\Psi_{l m}(\omega, r)=\frac{1}{2 \pi} \int d \Omega d t e^{i \omega t}{ }_{-2} S_{l m}^{*}(\theta, \phi)\left[r^{4} \delta \Psi_{4}(t, r, \theta, \phi)\right],
$$

where ${ }_{-2} S_{l m}(\theta, \phi)$ is the spin-weighted spherical harmonic of spin -2 . In terms of $Z_{l m}^{p o l}(\omega, r)$ and $Z_{l m}^{a x}(\omega, r), \quad \Psi_{l m}(\omega, r)$ is

$$
\begin{aligned}
\Psi_{l m}(\omega, r) & =\frac{r^{3} \sqrt{n(n+1)}}{4 \omega}\left[V^{a x} Z_{l m}^{a x}+\left(W^{a x}+2 i \omega\right) \Lambda_{+} Z_{l m}^{a x}\right] \\
& -\frac{r^{3} \sqrt{n(n+1)}}{4}\left[V^{p o l} Z_{l m}^{p o l}+\left(W^{p o l}+2 i \omega\right) \Lambda_{+} Z_{l m}^{p o l}\right]
\end{aligned}
$$

where $\quad 2 n=(l-1)(l+2), \quad \Lambda_{+}=\frac{d}{d r_{*}}+i \omega=\frac{\Delta}{r^{2}} \frac{d}{d r}+i \omega, \quad V^{\text {pol }} \quad$ and $\quad V^{a x}$ are the Zerilli and the Regge-Wheeler potentials [4], [5], and

$$
\begin{aligned}
W^{a x} & =\frac{2}{r^{2}}(r-3 M), \\
W^{p o l} & =2 \frac{n r^{2}-3 M n r-3 M^{2}}{r^{2}(n r+3 M)} .
\end{aligned}
$$

Outside the star we integrate the inhomogeneous Bardeen-Press-Teukolsky BPT equation 6.7]

$$
\left\{\Delta^{2} \frac{d}{d r}\left[\frac{1}{\Delta} \frac{d}{d r}\right]+\left[\frac{\left(r^{4} \omega^{2}+4 i(r-M) r^{2} \omega\right)}{\Delta}-8 i \omega r-2 n\right]\right\} \Psi_{l m}(\omega, r)=-T_{l m}(\omega, r),
$$

where $\Delta=r^{2}-2 M r$, and the source term $T_{l m}(\omega, r)$ is that appropriate to describe the point mass $m_{0}$ moving on a given orbit around the star. In Paper I we discussed how to construct the solution of eq. (2.4) in the general case of elliptic orbits. In this paper, since 
we are interested mainly in the last phases of the evolution of binary systems, when the orbit has already been circularized, we focus on circular orbits and give the explicit solution of the equations in that case. If the mass $m_{0}$ moves on an orbit of radius $R_{0}$ the geodesic equations give

$$
\bar{\gamma} \equiv \dot{t}=\frac{E}{1-\frac{2 M}{R_{0}}}, \quad \omega_{K} \equiv \frac{d \varphi}{d t}=\frac{\dot{\varphi}}{\bar{\gamma}}
$$

where the dot indicates differentiation with respect to proper time. $E$ is the energy of the particle per unit mass, and $\omega_{K}$ is the keplerian orbital frequency

$$
\omega_{K}=\sqrt{\frac{M}{R_{0}^{3}}} .
$$

The source term can be written as

$$
T_{l m}(\omega, r)=\delta\left(\omega-m \omega_{K}\right)\left[{ }_{0} S_{l m}^{*}\left(\frac{\pi}{2}, 0\right)_{0} U_{l m}+{ }_{-1} S_{l m}^{*}\left(\frac{\pi}{2}, 0\right){ }_{-1} U_{l m}+{ }_{-2} S_{l m}^{*}\left(\frac{\pi}{2}, 0\right)_{-2} U_{l m}\right] .
$$

The functions ${ }_{s} U_{l m}$ are given by

$$
\begin{aligned}
{ }_{0} U_{l m}= & \left(-2 \pi \sqrt{n(n+1)} \frac{\bar{\gamma} \Delta_{0}^{2}}{R_{0}^{2}}\right) \delta\left(r-R_{0}\right), \\
{ }_{-1} U_{l m}= & \delta\left(r-R_{0}\right)\left(2 \pi \sqrt{2 n} m \bar{\gamma} \omega_{K}^{2} \Delta_{0} R_{0}^{2}\right)-\delta^{\prime}\left(r-R_{0}\right) \frac{\Delta^{2}}{r^{2}}\left(2 \pi \mathrm{i} \sqrt{2 n} \bar{\gamma} \omega_{K} R_{0}^{2}\right), \\
{ }_{-2} U_{l m}= & \delta\left(r-R_{0}\right)\left[\mathrm{i} \pi m \omega_{K}^{3} \bar{\gamma} \frac{\Delta_{0}^{2}}{R_{0}^{2}}\left(\frac{r^{6}}{\Delta}\right)_{0}^{\prime}-\pi m^{2} \omega_{K}^{4} \bar{\gamma} R_{0}^{6}\right]+ \\
& +\delta^{\prime}\left(r-R_{0}\right)\left[\Delta r^{3}\left(2 \pi \mathrm{i} m \omega_{K}^{3} \bar{\gamma} R_{0}\right)+\Delta^{2}\left(4 \pi \omega_{K}^{2} \bar{\gamma} R_{0}\right)\right]+ \\
& +\delta^{\prime \prime}\left(r-R_{0}\right)\left[\Delta^{2} r\left(\pi \omega_{K}^{2} \bar{\gamma} R_{0}\right)\right],
\end{aligned}
$$

where the prime indicates differentiation with respect to $r$, and a subscript 0 means evaluation at $r=R_{0}$. As in Paper I, the solution of Eq. (2.4) which satisfies the boundary conditions of pure outgoing radiation at radial infinity, and matches continuously with the interior solution can be found by the Green's function technique, and the amplitude of the wave emerging at radial infinity can be shown to be (cf. Paper I, Eqs. (4.3)-(4.7))

$$
A_{l m}(\omega)=-\frac{1}{W_{l m}(\omega)} \int_{R}^{\infty} \frac{d r^{\prime}}{\Delta^{2}} \Psi_{l m}^{1}\left(\omega, r^{\prime}\right) T_{l m}\left(\omega, r^{\prime}\right)
$$

where $W_{l m}(\omega)$ is the Wronskian of the two independent solutions of the homogeneous BPT equation

$$
W_{l m}(\omega)=\frac{1}{\Delta}\left[\Psi_{l m}^{1} \Psi_{l m, r}^{0}-\Psi_{l m}^{0} \Psi_{l m, r}^{1}\right]
$$

and $\quad \Psi_{l m}^{0}$ and $\Psi_{l m}^{1}$ satisfy the following equations

$$
\left\{\begin{array}{l}
\mathbb{L}_{B P T} \Psi_{l m}^{0}(\omega, r)=0 \\
\Psi_{l m}^{0}(\omega, r \rightarrow \infty)=r^{3} e^{\mathrm{i} \omega r_{*}}
\end{array}, \quad\left\{\begin{array}{l}
\mathbb{L}_{B P T} \Psi_{l m}^{1}(\omega, r)=0 \\
\Psi_{l m}^{1}(\omega, R)=\bar{\Psi}_{l m}(\omega, R) \\
\Psi_{l m}^{1}(\omega, R)=\bar{\Psi}_{l m}^{\prime}(\omega, R)
\end{array},\right.\right.
$$


where $\mathbb{L}_{B P T}$ is the differential operator on the left hand side of the BPT equation, and $\bar{\Psi}_{l m}(\omega, R)$ is the function constructed according to Eq. (2.3), in terms of the perturbed functions computed in the interior, and evaluated at the surface of the star. The integral in Eq. (2.8) can explicitly be evaluated by making a repeated use of the following property of the $\delta$-function

$$
\int g(r) \partial_{r}\left[f(r) \delta\left(r-R_{0}\right)\right] d r=-g^{\prime}\left(R_{0}\right) f\left(R_{0}\right)
$$

The result is

$$
\begin{aligned}
A_{l m}(\omega) & =-m_{0} \frac{\delta\left(\omega-m \omega_{K}\right)}{W_{l m}(\omega)}\left\{\Psi _ { l m } ^ { 1 } ( \omega , R _ { 0 } ) \left[{ }_{0} S_{l m}^{*}\left(-2 \pi \sqrt{n(n+1)} \frac{\bar{\gamma}}{R_{0}^{2}}\right)\right.\right. \\
& +{ }_{-1} S_{l m}^{*}\left(2 \pi \sqrt{2 n} m \bar{\gamma} \omega_{K}^{2} \frac{R_{0}^{2}}{\Delta_{0}}-4 \pi \mathrm{i} \sqrt{2 n} \bar{\gamma} \omega_{K} \frac{1}{R_{0}}\right) \\
& \left.+{ }_{-2} S_{l m}^{*}\left(2 \mathrm{i} \pi m \omega_{K}^{3} \bar{\gamma} \frac{R_{0}^{4}}{\Delta_{0}^{2}}\left(R_{0}-M\right)-\pi m^{2} \omega_{K}^{4} \bar{\gamma} \frac{R_{0}^{6}}{\Delta_{0}^{2}}\right)\right] \\
& +\Psi_{l m}^{1^{\prime}}\left(\omega, R_{0}\right)\left[{ }_{-1} S_{l m}^{*}\left(2 \pi \mathrm{i} \sqrt{2 n} \bar{\gamma} \omega_{K}\right)+{ }_{-2} S_{l m}^{*}\left(-2 \pi \mathrm{i} m \omega_{K}^{3} \bar{\gamma} \frac{R_{0}^{4}}{\Delta_{0}}-2 \pi \omega_{K}^{2} \bar{\gamma} R_{0}\right)\right] \\
& \left.+\Psi_{l m}^{1^{\prime \prime}}\left(\omega, R_{0}\right){ }_{-2} S_{l m}^{*}\left(\pi \omega_{K}^{2} \bar{\gamma} R_{0}^{2}\right)\right\} .
\end{aligned}
$$

If we define

$$
A_{l m}(\omega)=\hat{A}_{l m}(\omega) \delta\left(\omega-m \omega_{K}\right),
$$

the time-averaged energy-flux

$$
\dot{E}^{R} \equiv\left\langle\frac{d E_{G W}}{d t}\right\rangle=\lim _{T \rightarrow \infty} \frac{E_{G W}}{T}=\lim _{T \rightarrow \infty} \frac{1}{T} \sum_{l m} \int d \omega\left(\frac{d E_{G W}}{d \omega}\right)_{l m}
$$

can be written in terms of $\hat{A}_{l m}(\omega)$ as follows

$$
\dot{E}^{R}\left(m \omega_{K}\right)=\sum_{l m} \frac{1}{4 \pi\left(m \omega_{K}\right)^{2}}\left|\hat{A}_{l m}\left(m \omega_{K}\right)\right|^{2} \equiv \sum_{l m} \dot{E}_{l m}^{R}
$$

In order to evaluate $\Psi_{l m}^{0}$ and $\Psi_{l m}^{1}$, the BPT equation is integrated with the same adaptive Runge-Kutta used for the equations in the interior. We must remark that close to a resonance the solutions need to be computed very accurately, since the Wronskian is the difference between two terms that almost cancel each other. When required, the tolerance in the integration is decreased until convergence is reached. Typically, computing the power emitted at a given orbit takes about $10 \mathrm{~s}$ on a PC with a Pentium IV $1 \mathrm{GHz}$ processor.

Since the orbital frequency is related to the orbital velocity $v$ and to the semilatus rectum $p=R_{0} / M$ by the following relations

$$
v=\left(M \omega_{K}\right)^{1 / 3}=\frac{1}{\sqrt{p}}
$$

the energy flux $\dot{E}^{R}$ can also be considered as a function of $v$ or $p$.

In the following, we shall normalize $\dot{E}^{R}$ to the Newtonian quadrupole energy flux

$$
\dot{E}^{N}=\frac{32}{5} \frac{m_{0}^{2}}{M^{2}} v^{10}
$$




\section{COMPARING THE GRAVITATIONAL FLUX OF STARS AND BLACK HOLES}

In our analysis, we consider five models of stars with a polytropic EOS, using two values of the polytropic index, $n=1$ and $n=1.5$. The values of the central density, the ratio $\alpha_{0}$ between the energy density and the pressure at the centre, the mass, the radius

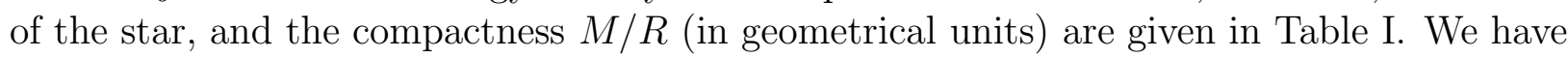
chosen polytropic models for simplicity, but without loss of generality, because the differences in the gravitational flux are expected to depend more on global properties such as mass, radius, average density, or compactness, rather than on the specific matter distribution. The parameters we choose encompass a reasonable range of stellar models (radius ranging from 9 to $15 \mathrm{~km}$ ), and the polytropic exponents, $\Gamma \equiv 1+1 / n=5 / 3$ and $\Gamma=2$, cover most of the range of structural properties obtained with realistic EOS's.

\section{A. Power radiated and resonant modes}

For each model, we integrate the equations of stellar perturbations as described in Sec. II, assuming that a point like mass, $m_{0}$, is moving on a circular orbit of radius $R_{0}$ with orbital velocity $v$, and we compute the energy flux emitted in gravitational waves normalized to the Newtonian quadrupole energy flux, $P \equiv \dot{E}^{R} / \dot{E}^{N}$, where $\dot{E}^{R}$ and $\dot{E}^{N}$ are given in Eqs. (2.14) and (2.16). In Ref. 8 10] the gravitational emission of a Schwarzschild black hole perturbed by a massive particle in circular orbit was studied numerically in great detail. Our results for the black hole agree with them with an accuracy of (at least) one part in $10^{6}$. In order to allow an easy comparison, and for future reference, we show in Table [I] the contribution of the $2 \leq l \leq 5$ multipoles to the total gravitational power. The results for the black hole can directly be compared to Table II in Ref. [10].

In Fig. 1 we plot the normalized energy flux, $P(v)$, as a function of the orbital velocity, for the models of star we have considered, and for the black hole. $P(v)$ has been obtained by adding the contributions of different l's and $m$ 's, with $2 \leq l \leq 7$. As discussed in paper I, a multipole of order $l$ contributes to the total power as a correction of order $p^{2-l}$. By truncating our multipole expansion at $l=7$ we are incurring in a relative error of order $p^{-6}=v^{12}$. It should be mentioned that $P(v)$ is independent of the mass $m_{0}$. For the black hole and for the stellar model $\mathrm{D}$, the plots extend up to the velocity $v=0.408$, which corresponds to the Innermost Stable Circular Orbit (ISCO), $R_{0}=6 M$; for model A, $\mathrm{B}$ and $\mathrm{C}$ the ISCO would be in the interior of the star, therefore the plots are truncated at a smaller velocity, which corresponds to $m_{0}$ reaching the stellar surface. Sharp peaks appear if the central object is a star: they correspond to the excitation of the fundamental quasi-normal modes of the star for different values of the harmonic index $l$. In the case of model B the first p-mode for $l=2$ is also excited. In Table III we show the values of the radius $R_{0}$, of the dimensionless orbital velocity $v$, and of the keplerian frequency $\nu_{K}$ of the orbit that corresponds to the excitation of the fundamental modes of the star for different l's for the considered stellar models. The corresponding frequencies of the $f$-mode are given in the last column. From the analytical form of the stress-energy tensor (2.7), it is easy to see

that, for each assigned $l$, a mode of the star is excited when the orbital frequency satisfies the resonant condition 


$$
m \nu_{K}=\nu_{i}
$$

where $\nu_{i}$ is the mode frequency. Table III shows that the frequency of the f-mode increases with $l$; however, eq. (3.1) shows that, for instance, the orbital velocity that corresponds to the excitation of the $f$-mode for $l=3$ is lower than that needed to excite the $f$-mode for $l=2$; this means that in the process of coalescence of the "binary system" formed by the star and the point mass $m_{0}$, the $f$-mode for $l=3$ is excited before the $l=2 f$-mode, and similarly the $l=4 \mathrm{f}$-mode is excited before that for $l=3$, while the gravitational wave frequency is higher. The peaks corresponding to higher $l$ are narrower, so that peaks for $l>4$ are difficult to locate, even with the use of an extremely refined grid. From newtonian theory we know that the $f$-mode frequency scales with the square root of the average density of the star. This is true also in general relativity and the dependency on $\sqrt{M / R^{3}}$ is still linear [11]. This explains why for a chosen value of the polytropic index $n$ the peaks for more compact stars occur at higher frequency, i.e. at higher $v$, (for instance, compare in Fig. 11 the two curves for model $\mathrm{B}$ and $\mathrm{D}$, for which $n=1$ and $R=15 \mathrm{~km}$ and $R=9.8$ $\mathrm{km}$, respectively). Since the peaks corresponding to the mode excitation are very high, the scale chosen on the vertical axis of Fig. 1 makes the response of the black hole to appear as a flat line. The reason is that, since the frequency of the lowest quasi-normal modes of a black hole are higher than those of a star with the same mass, the circular orbit that would excite them would have a radius smaller than $6 M_{B H}$. Thus, in the range of $v$ considered in Fig. 11 the energy flux emitted by the black hole is due essentially to the orbital motion. In Fig. 2, we show a zoom of Fig. 1 1 restricted to the region $v<0.28$, which is far enough from the resonant orbits (except that for model A). In this case we can appreciate the differences between the emission of different stellar models and that of a black hole. If the orbital velocity is smaller than 0.16 all curves are practically indistinguishable.

Fig. 2 shows that the normalized energy fluxes emitted by different stellar models have a different slope, and are always larger than the flux emitted by the black hole. The curve for model $\mathrm{E}\left(n=1.5, R_{0}=9 \mathrm{~km}\right)$ is practically indistinguishable from the black hole curve; that for model $\mathrm{D}\left(n=1, R_{0}=9.8 \mathrm{~km}\right)$ is also very close to the black hole result. At first sight we may relate this behavior to the fact that these stars are more compact than models A,B,C; however, the steepest raise of the curves of models A,B,C could also be a marginal effect of the resonances, or may be due to a different coupling between the orbital motion and the stellar structure.

\section{B. A harmonic oscillator model}

In order to better understand the underlying physical picture, we shall use a toy model which has been employed in many contexts to study the effects of stellar resonances (see for instance Ref. [12]). Since a star oscillating in a quasi-normal mode of complex frequency $\omega_{0}-i \omega_{i}$ emits a gravitational wave of amplitude $\sim C e^{-i\left(\omega_{0}-i \omega_{i}\right) t}$, it can be modeled as a harmonic oscillator which satisfies the differential equation $\ddot{X}+2 \omega_{i} \dot{X}+\left(\omega_{0}^{2}+\omega_{i}^{2}\right) X=0$. Here the amplitude $X$ is assumed to be normalized to some reference amplitude, for instance to the amplitude of the newtonian quadrupole, $A^{N}$, which we define in terms of the newtonian energy flux of Eq. (2.16) as follows 


$$
\dot{E}^{N}=\frac{1}{4 \pi \omega^{2}}\left|A^{N}(\omega)\right|^{2}
$$

The particle orbiting the star acts as a driving force proportional to $\omega^{2} e^{-i \omega t}$, and the amplitude of the forced oscillation is found by solving the inhomogeneous equation

$$
\ddot{X}+2 \omega_{i} \dot{X}+\left(\omega_{0}^{2}+\omega_{i}^{2}\right) X=b \omega^{2} e^{-i \omega t} ;
$$

the solution can be written in the form $X(t)=X(\omega) e^{-i \omega t}$, where

$$
X(\omega)=\frac{-b \omega^{2}}{\omega^{2}-\omega_{0}^{2}-\omega_{i}^{2}+2 i \omega_{i} \omega} .
$$

We shall now assume that, near a resonance, the total amplitude of the wave emitted by the perturbed star is the sum of two contributions, one due to the orbital motion, the other to the star pulsating in its quasi-normal mode, i.e.

$$
A_{t o t}(\omega)=A^{N}(\omega)[1+X(\omega)]
$$

According to this model, the normalized energy flux $P_{\text {res }}$ can be written as

$$
P_{\text {res }}(\omega)=\frac{\left|A_{t o t}\right|^{2}}{\left|A^{N}\right|^{2}}=|1+X(\omega)|^{2}=\frac{\left[(1-b) \omega^{2}-\omega_{0}^{2}-\omega_{i}^{2}\right]^{2}+\left[2 \omega_{i} \omega\right]^{2}}{\left[\omega^{2}-\omega_{0}^{2}-\omega_{i}^{2}\right]^{2}+\left[2 \omega_{i} \omega\right]^{2}} .
$$

$P_{\text {res }}(\omega)$ has a maximum in $\omega_{0}$ and a minimum in $\frac{\omega_{0}}{\sqrt{1-b}}$; the height of the maximum is $\frac{b^{2} \omega_{0}^{2}}{4 \omega_{i}^{2}}$ and that of the minimum is $\frac{4 \omega_{i}^{2}(1-b)}{b^{2} \omega_{0}^{2}}$ (up to corrections of order $O\left(\left(\omega_{i} / \omega_{0}\right)^{4}\right)$ ). For $\omega<<\omega_{0}, P_{\text {res }}(\omega)$ tends to 1 , while after the resonance it tends to $(1-b)$. In order to check how good is the harmonic oscillator model to describe the behavior of the star near a resonance, the quantity $P_{\text {res }}(\omega)$ has to be compared with the normalized energy flux, $P(\omega)$, computed by integrating the equations of stellar perturbations. In Fig. 3 we plot $P_{\text {res }}(\omega)$ (continuous line) and the numerically evaluated $P_{l=2, m=2}(\omega)$ (diamonds) as a function of $\omega M$, in a frequency region near the fundamental mode for $l=2, m=2$, for the stellar model $\mathrm{C}$. The parameter $b$ is found from the locations of the maximum and the minimum, i.e. $b=1-\left(\omega_{\max } / \omega_{\min }\right)^{2}$, and $\omega_{i}$ is chosen in such a way that the amplitude of the maxima of the two functions coincide. The results are in very good agreement (relative differences are of order $10^{-5}$ ) except very close to the minimum, where the function $P_{l=2, m=2}(\omega)$ goes to zero. Incidentally, it should be mentioned that the evaluation of the exact value of the minimum for each $l$ is not so important, as far as the total energy flux is concerned; indeed, the total flux $P(\omega)$, evaluated at the frequency which corresponds to a minimum for a given $l$, has a finite value, because it is the sum of contributions from different $l$ 's and $m$ 's, and it is dominated by the other multipoles.

The simple analytical model works surprisingly well in the whole range of each resonance, capturing the parabolic behavior in the region $\left|\omega-\omega_{0}\right|^{2} \ll 1$, used by several authors to calculate damping times, and also describing the global behavior of the normalized flux with high accuracy. We found that the same procedure can satisfactorily be applied to the other stellar models, and to resonances corresponding to higher values of $l$. The values of the 
frequencies where the maximum and the minimum occur, the forcing amplitude $b$ and the imaginary part of the frequency of the fundamental mode, $\omega_{i}$, are summarized in Table IV for the five stellar models. Note that the $\omega_{i} M$ scales approximately as $10^{-2 l}$, and $b$ as $10^{-l}$.

The agreement between the numerical result and the toy model suggests the following question: can the effects of a resonance be subtracted from the global signal ? The answer is shown in Fig. 4 , where we plot the $(l=2, m=2)$ contribution to the flux, $P(v)_{l=2, m=2}$, with a solid line, and the result of subtracting the effect of the resonance with dashed lines, versus the orbital velocity. The resonance-free curve has been obtained dividing the normalized energy flux emitted by the perturbed star by the model given in (3.6), i.e. $P(v)_{l=2, m=2} / P_{\text {res }}(v)$. Now the question of whether or not the different slope of the curves plotted in Fig. 2 can be attributed to the effect of resonant excitation of a stellar quasinormal mode can be answered positively. The increase in the energy output at orbital velocities of about $v=0.2$ is just an effect of the resonance tail. However, it is not the only effect. Indeed, since we are normalizing the emitted flux to the newtonian quadrupole flux, if the signal emitted by the perturbed star would be, as we assume in eq. (3.5), the sum of a term due to the orbital motion (the quadrupole) and a term due to the resonance, the dashed line in Fig. 1 should be a horizontal straight line. Conversely, it is a slightly decreasing function of $v$. In order to see if this is a general feature, we have also calculated $P(v)_{l=2, m=2} / P_{r e s}(v)$ for the stellar models A, B and D. The results are plotted in Fig. 5. For comparison, we also plot $P(v)_{l=2, m=2}$ for a black hole and for the stellar model E, computed without subtracting the contribution of the resonances. The reason is that we cannot subtract this contribution because, as mentioned before, black hole quasi-normal modes cannot be excited before the ISCO and similarly, to excite the f-mode of model E the point mass should move on an orbit with radius smaller than the stellar radius. We see that after the subtraction there is still a difference between different EOS's; in particular, if we classify the models A,B,C,D according to their stiffness (where a stiffness indicator is, for example, the speed of sound at a given density), we find that the stiffer the EOS is, the smaller is the orbital contribution to the total emission. We must remark, however, that when the effect of the resonances is included, this trend is inverted; stiffer models emit more energy, because their resonant frequencies are smaller and their effect is more pronounced.

The influence that the structural effects discussed above may have on the detection of these signals by terrestrial detectors, should be estimated by computing the phase evolution of the signal; this can be done by taking into account the evolution of the orbit due to radiation reaction effects. Although this problem will be specifically addressed in a separate paper, we can anticipate that, depending on the stellar model, most of the phase shift is accumulated during the last 50 cycles before merging; this effect is not likely to be detectable by the first generation of LIGO and VIRGO detectors, unless we are so lucky that a NSNS coalescence occurs closer than $10 \mathrm{Mpc}$. However, the situation would be different if a detector like EURO [13, recently proposed, would be constructed, because it would be very sensitive in the frequency range (600-1200) $\mathrm{Hz}$ where the structural effects become significant. Quantifying precisely the differences between different signals and templates is out of the scope of this paper, and requires a careful calculation of the overlaps of the "true signal" with those that are currently used as templates. This work is in progress and will be reported in a forthcoming paper. 


\section{ON THE VALIDITY OF THE PERTURBATIVE APPROACH}

The theory of perturbations of black holes excited by a point particle developed since the early seventies was based on the assumption that the mass of the particle is much smaller than the black hole mass; under this condition, the effect of the particle on the black hole is that of inducing a small perturbation on the equilibrium configuration, and the stress energy tensor of the mass $m_{0}$ can be considered as a source for the perturbed equations. In addition, since $m_{0}$ does not affect the background geometry, it will move on a geodesic of the unperturbed spacetime. The same assumption can be done to study the perturbations of a star, as we do in this paper. However, since one of the purposes of our study is to have an insight on the last phases of the coalescence of a true binary system composed of stars of comparable mass, we may ask the following question: how big can the mass $m_{0}$ be, in order the deviation it induces on the gravitational field and on the thermodynamical structure of the companion star to be considered as a perturbation? Or, we can formulate the same question in a different way: given a mass $m_{0}$, not necessarily small, moving on a circular orbit around a star, up to which distance its effect on the companion can be considered as a perturbation? I To answer this question in Fig. 6 we plot the fluid and the gravitational perturbed functions inside the star, for $l=2, m=2$ (the most significant contribution) and for the stellar model D, assuming that the point mass is moving on a circular orbit at a distance $R_{0}=3 R$ from the star. In the upper panel we plot the radial component of the Lagrangian displacement normalized to the stellar radius and the Lagrangian perturbations of the density and of the pressure normalized to their equilibrium values. In the lower panel we plot the perturbations of the metric $2 N(r)$ and $2 L(r)$, that have to be compared to unity (cf. Paper I, Eq. (2.1)). All quantities are normalized to the ratio $m_{0} / M$. Similar results are obtained for the other stellar models. Fig. 6 shows that, even if we assume that the two masses are equal, all perturbations belonging to the fluid or to the gravitational field are small compared to the corresponding unperturbed functions. Thus, the perturbative approach holds even when the two bodies have comparable mass and are as close as three stellar radii. Corrections of the same order of magnitude have been found in full nonlinear stationary solutions of binary neutron stars [14]

\section{CONCLUDING REMARKS}

In this paper we have studied in a full general relativistic approach how the internal structure of a neutron star affects its gravitational emission when the star is perturbed by a close, orbiting companion. Our study has been done by using a perturbative approach in the frequency domain, and the massive object which perturbs the star has been assumed to be a point particle of mass $m_{0}$. We have considered five models of star with global properties - like compactness, average density, stiffness of the EOS - that encompass a wide range of

1 We neglect the fact that if $m_{0}$ is large it will not move on a geodesic of the unperturbed spacetime. This point will be discussed in the concluding remarks. 
stellar properties, and we have compared their behavior with that of a black hole excited by the same source.

The results we find can be summarized as follows. The internal structure of the star affects the emitted flux of gravitational waves only when the orbital velocity of $m_{0}$ is (approximately) higher than one fifth of the speed of light, i.e. when $m_{0}$ is very close to the central star. If the mass of the star and $m_{0}$ were comparable and about $1.4 M_{\odot}$, this velocity would correspond to an emission frequency $\gtrsim 185 \mathrm{~Hz}$. For lower values of $v$ (larger orbital separation) neutron stars and black holes behave in the same manner and the gravitational fluxes they emit are practically indistinguishable.

The difference in the energy flux emitted by a star and a black hole arises mainly because of the excitation of the fundamental mode of the star for different $l$ 's, which can be modeled extremely well in terms of a suitably defined harmonic oscillator. The results for model A suggest that the emission properties of low mass neutron star binary systems would deviate significantly from those of black hole-black hole binary systems.

Once the effect of the resonant excitation of the quasi-normal modes of the star are subtracted, we find that there is still a residual difference between the emission of neutron stars and black holes, and that stars with stiffer EOS emit less energy than a black hole. However, this trend is inverted when the effect of resonances is included.

It should be stressed that the region where corrections due to the EOS begin to be significant is the same region where high order Post Newtonian (PN) corrections play a significant role. Thus these effects should be included in constructing templates to be used in the data analysis of gravitational detectors. In addition, the corrections introduced by the tail of the resonant f-mode is comparable, if not larger, to that introduced by the high order PN corrections [15].

The problem of mode excitation during the latest phases of the evolution of a binary system, and the consequences it has on the emitted gravitational signal, have previously been studied by Ho \& Lai [16] by a newtonian approach. In particular, as far as the excitation of the f-mode is concerned, they consider the case of a rotating, incompressible model of star, which is very close to our model E, whereas we use a relativistic perturbative approach and focus on the differences which may arise because of different EOS's. Apart from this, we basically reach similar conclusions about the order of magnitude of the resonant effects and on the regime where they may play a role.

It is interesting to discuss to what extent can we extrapolate our results to the case when the mass $m_{0}$ is comparable to that of the central star, i.e, to simulate a true coalescing binary system. Indeed, the results of Sec. IV indicate that the perturbative approach holds also when two bodies of comparable mass are very close, even closer than 3 stellar radii. A naive generalization of the energy fluxes we obtain could be that of rescaling the amplitude of the energy flux by the appropriate value of $m_{0}$, and to change the orbital frequency $\omega_{K}=\sqrt{M / R_{0}^{3}}$ (and consequently the emission frequency $\omega_{G W}=m \omega_{K}$ ) by replacing the mass of the star $M$ with the total mass of the system $M_{t}=M+m_{0}$; it is worth mentioning that, in this case, given a certain orbital radius $R_{0}$, the orbital frequency corresponding to that radius would be higher than that of the point particle on the same orbit. This can easily be done, but the results should be considered only as an indication of what may happen in reality. Indeed, in order to correctly generalize the results, the perturbative approach should be improved in many ways: the geodesic equation which are used to describe the motion of 
$m_{0}$ should be replaced by the correct equations of motion of a two-body system, as seen in the coordinate system centered on one star; these equations are now known at the 3.5 PN level [17], and they would change the stress energy tensor we put on the right-hand side of our equations; the problem of chosing the reference frame in which to compute the radiated power as seen by a distant observer is a very delicate one, and in order to compare the results of the perturbative approach for equal masses with those of the post-newtonian approach one should change to a frame centered in the center of mass of the binary system; in computing the orbital evolution due to radiation reaction effects it is customary to use the adiabatic approximation which assumes that the timescale of the orbital evolution is larger than the orbital period; we think that the role of this assumption has to be investigated very carefully during the last few cycles before coalescence. All of these problems are important: we are looking for very small effects which arise just before coalescence, and they may introduce corrections of a few percents in the emitted power, which are of the same order as the effects of stellar structure we discuss in this paper. Finally, one should certainly consider rotating stars. Rotation may change the situation in a positive direction, because we know that it has the effect of lowering some of the mode frequencies, and this may enhance the marginal effect of the mode excitation which we see in the tail of the signals emitted by compact stars.

In future papers we plan to implement all these effects in our perturbative approach, with the final goal of providing accurate templates which may be used in the data analysis of future high sensitive detectors operating in the $\mathrm{kHz}$ region.

\section{ACKNOWLEDGMENTS}

We are indebted to the anonymous referee for a number of useful comments and suggestions. This work has been supported by the EU Programme 'Improving the Human Research Potential and the Socio-Economic Knowledge Base' (Research Training Network Contract HPRN-CT-2000-00137). 


\section{TABLES}

TABLE I. Parameters of the polytropic stars we consider in our analysis: the polytropic index $n$, the central density, the ratio $\alpha_{0}=\epsilon_{0} / p_{0}$ of central energy density to central pressure, the mass, the radius and the ratio $M / R\left(\alpha_{0}\right.$ and $M / R$ are in geometric units). The central energy density is chosen in such a way that the stellar mass is equal to $1.4 M_{\odot}$, except for model $\mathrm{A}$, the mass of which is about one solar mass.

\begin{tabular}{cllllll}
\hline \hline Model number & $n$ & $\rho_{c}\left(\mathrm{~g} / \mathrm{cm}^{3}\right)$ & $\alpha_{0}$ & $M\left(M_{\odot}\right)$ & $R(\mathrm{~km})$ & $M / R$ \\
\hline $\mathrm{A}$ & 1.5 & $1.00 \times 10^{15}$ & 13.552 & 0.945 & 14.07 & 0.099 \\
$\mathrm{~B}$ & 1 & $6.584 \times 10^{14}$ & 9.669 & 1.4 & 15.00 & 0.138 \\
$\mathrm{C}$ & 1.5 & $1.260 \times 10^{15}$ & 8.205 & 1.4 & 15.00 & 0.138 \\
$\mathrm{D}$ & 1 & $2.455 \times 10^{15}$ & 4.490 & 1.4 & 9.80 & 0.211 \\
$\mathrm{E}$ & 1.5 & $8.156 \times 10^{15}$ & 2.146 & 1.4 & 9.00 & 0.230 \\
\hline \hline
\end{tabular}

TABLE II. Decomposition of the gravitational luminosity $\left(M / m_{0}\right)^{2} \dot{E}^{R}$ into multipole contributions for $p=10$. The BH results can be compared directly to those of Table II in Ref. [10].

\begin{tabular}{|c|c|c|c|c|c|c|c|}
\hline$l$ & $m$ & $\mathrm{BH}$ & $\mathrm{A}$ & B & $\mathrm{C}$ & $\mathrm{D}$ & $\mathrm{E}$ \\
\hline & 2 & $0.536879(-4)$ & $0.339696(-04)$ & $0.220321(-06)$ & $0.667680(-04)$ & $0.542028(-04)$ & $0.537526(-04)$ \\
\hline & 1 & $0.193161(-6)$ & $0.194489(-06)$ & $0.193693(-06)$ & $0.193491(-06)$ & $0.193244(-06)$ & $0.193189(-06)$ \\
\hline 8 & 3 & $0.642607(-5)$ & $0.588917(-05)$ & $0.599186(-05)$ & $0.564656(-05)$ & $0.643160(-05)$ & $0.642658(-05)$ \\
\hline & 2 & $0.479591(-7)$ & $0.481018(-07)$ & $0.479889(-07)$ & $0.479742(-07)$ & $0.479605(-07)$ & $0.479594(-07)$ \\
\hline & 1 & $0.571489(-9)$ & $0.712057(-09)$ & $0.587559(-09)$ & $0.577923(-09)$ & $0.571763(-09)$ & $0.571520(-09)$ \\
\hline & 4 & $0.953958(-6)$ & $0.918437(-06)$ & $0.944728(-06)$ & $0.948295(-06)$ & $0.954066(-06)$ & $0.953966(-06)$ \\
\hline & 3 & $0.877874(-8)$ & $0.879191(-08)$ & $0.878020(-08)$ & $0.877939(-08)$ & $0.877878(-08)$ & $0.877876(-08)$ \\
\hline 4 & 2 & $0.526223(-9)$ & $0.116026(-08)$ & $0.532315(-09)$ & $0.528287(-09)$ & $0.526252(-09)$ & $0.526227(-09)$ \\
\hline & 1 & $0.145758(-12)$ & $0.145966(-12)$ & $0.145781(-12)$ & $0.145768(-1$ & $0.145759(-12)$ & $0.145759(-12)$ \\
\hline & 5 & $0.152415(-6)$ & $0.149988(-06)$ & $0.152100(-06)$ & $0.152269(-06)$ & $0.152418(-06)$ & $0.152415(-06)$ \\
\hline & 4 & $0.149211(-8)$ & $0.149338(-08)$ & $0.149219(-08)$ & $0.149214(-08)$ & $0.149212(-08)$ & $0.149212(-08)$ \\
\hline & 3 & $0.182910(-9)$ & $0.168856(-09)$ & $0.184547(-09)$ & $0.183313(-09)$ & $0.182911(-09)$ & $0.182910(-09)$ \\
\hline & 2 & $0.381934(-12)$ & $0.382239(-12)$ & $0.381952(-12)$ & $0.381942(-12)$ & $0.381935(-12)$ & $0.381935(-12)$ \\
\hline 5 & 1 & $0.236763(-15)$ & $0.252126(-15)$ & $0.237281(-15)$ & $0.236943(-15)$ & $0.236765(-15)$ & $0.236764(-15)$ \\
\hline
\end{tabular}


TABLE III. In this table we give the values of the radius, orbital velocity and keplerian frequency $\left(\nu_{K}\right)$ of the circular orbits which correspond to the excitation of the fundamental mode of the considered stars for the first relevant multipoles, whose frequency is given in the last column. For model $\mathrm{E}$ we do not give these data for $l=2$ because in order to excite the corresponding mode $R_{0}$ should be smaller than the ISCO $(6 M)$.

\begin{tabular}{llllll}
\hline \hline Model & $l$ & $R_{0}(\mathrm{~km})$ & $v$ & $\nu_{K}(\mathrm{~Hz})$ & $\nu_{f}(\mathrm{~Hz})$ \\
\hline $\mathrm{A}$ & 4 & 31.8 & 0.255 & 567 & 2260 \\
& 3 & 29.0 & 0.267 & 651 & 1953 \\
& 2 & 26.0 & 0.282 & 767 & 1534 \\
\hline $\mathrm{B}$ & 3 & 22.9 & 0.300 & 626 & 1879 \\
& 2 & 21.0 & 0.313 & 711 & 1422 \\
\hline $\mathrm{C}$ & 3 & 21.2 & 0.312 & 702 & 2105 \\
& 2 & 18.9 & 0.331 & 835 & 1671 \\
\hline $\mathrm{D}$ & 3 & 15.5 & 0.365 & 1119 & 3358 \\
& 2 & 14.1 & 0.383 & 1296 & 2593 \\
\hline $\mathrm{E}$ & 3 & 13.5 & 0.391 & 1379 & 4138 \\
\hline \hline
\end{tabular}

TABLE IV. Parameters of the resonances (3.6) corresponding to the fundamental mode of the most relevant $l$-multipoles. All frequencies are normalized to the mass of the corresponding star.

\begin{tabular}{llllll}
\hline \hline Model & $l$ & $(\omega M)_{\max }$ & $(\omega M)_{\min }$ & $b$ & $\omega_{i} M$ \\
\hline $\mathrm{A}$ & 4 & 0.066218 & 0.066239 & 0.000645 & $9.67 \times 10^{-10}$ \\
& 3 & 0.057314 & 0.057495 & 0.006272 & $1.32 \times 10^{-7}$ \\
& 2 & 0.044823 & 0.046273 & 0.061682 & $1.81 \times 10^{-5}$ \\
\hline $\mathrm{B}$ & 3 & 0.081420 & 0.081645 & 0.005618 & $1.93 \times 10^{-7}$ \\
& 2 & 0.061581 & 0.063363 & 0.055367 & $1.90 \times 10^{-5}$ \\
\hline $\mathrm{C}$ & 3 & 0.091210 & 0.091402 & 0.004208 & $2.09 \times 10^{-7}$ \\
& 2 & 0.072299 & 0.074036 & 0.046373 & $2.47 \times 10^{-5}$ \\
\hline $\mathrm{D}$ & 3 & 0.145709 & 0.145895 & 0.002555 & $6.17 \times 10^{-7}$ \\
& 2 & 0.112454 & 0.114287 & 0.031836 & $5.77 \times 10^{-5}$ \\
\hline $\mathrm{E}$ & 3 & 0.179606 & 0.1796935 & 0.000971 & $4.91 \times 10^{-7}$ \\
\hline \hline
\end{tabular}




\section{FIGURES}

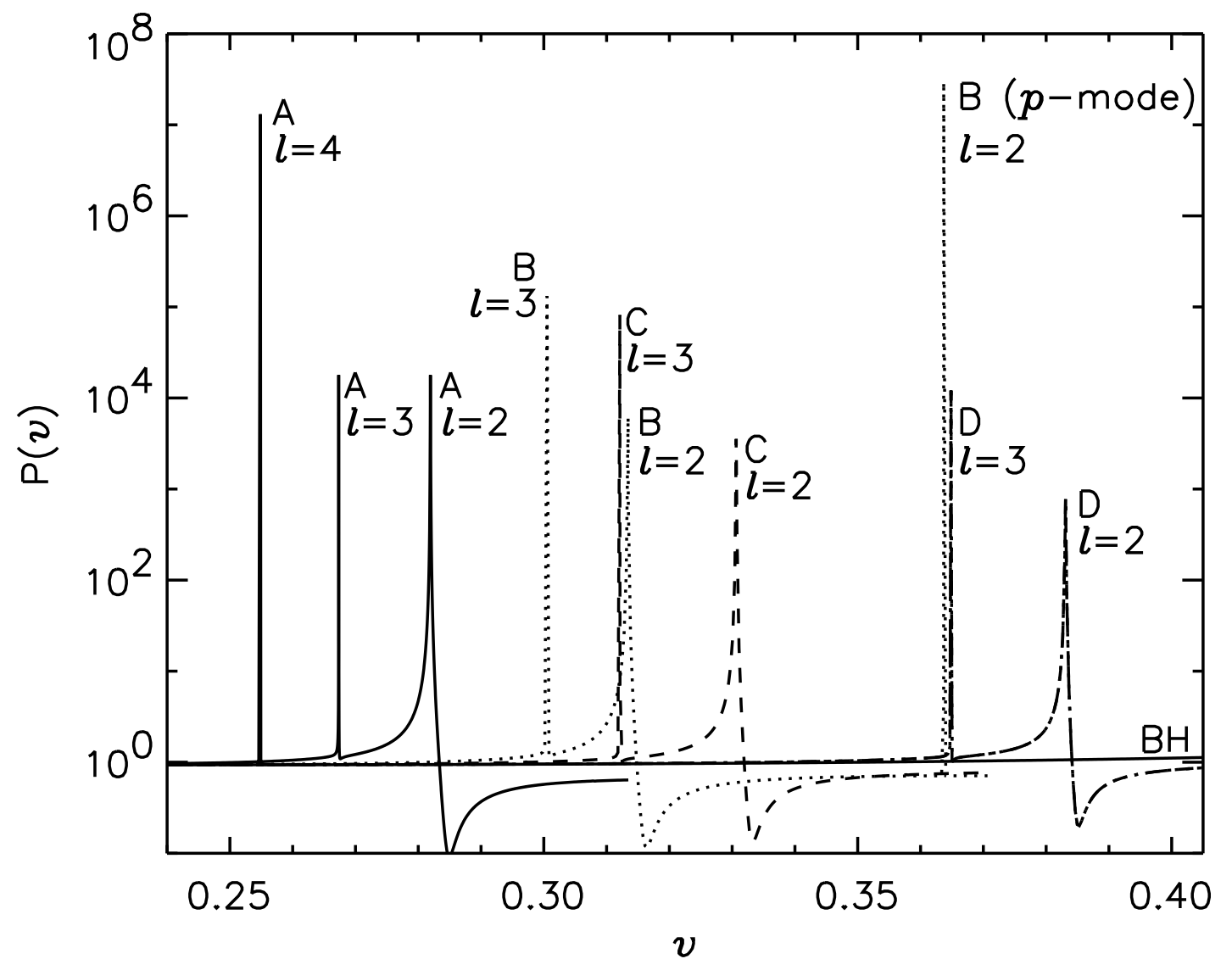

FIG. 1. The normalized energy flux, $P(v)$, is plotted as a function of the orbital velocity for the stellar models given in Table 1 and for a black hole. For model D and for the black hole the curves extend up to the velocity which correspond to the ISCO, whereas for the other models they stop when the mass $m_{0}$ reaches the surface of the star. The sharp peaks indicate that, for different values of the harmonic index $l$, the fundamental quasi-normal modes of the star are excited if the orbital frequency satisfies the resonant condition (3.1); the curve of the stellar model B has a peak at high $v$ which correspond to the excitation of the first p-mode for $l=2$. The most compact model $\mathrm{E}$ is not shown in the figure because at this scale it is indistinguishable from the black hole. 


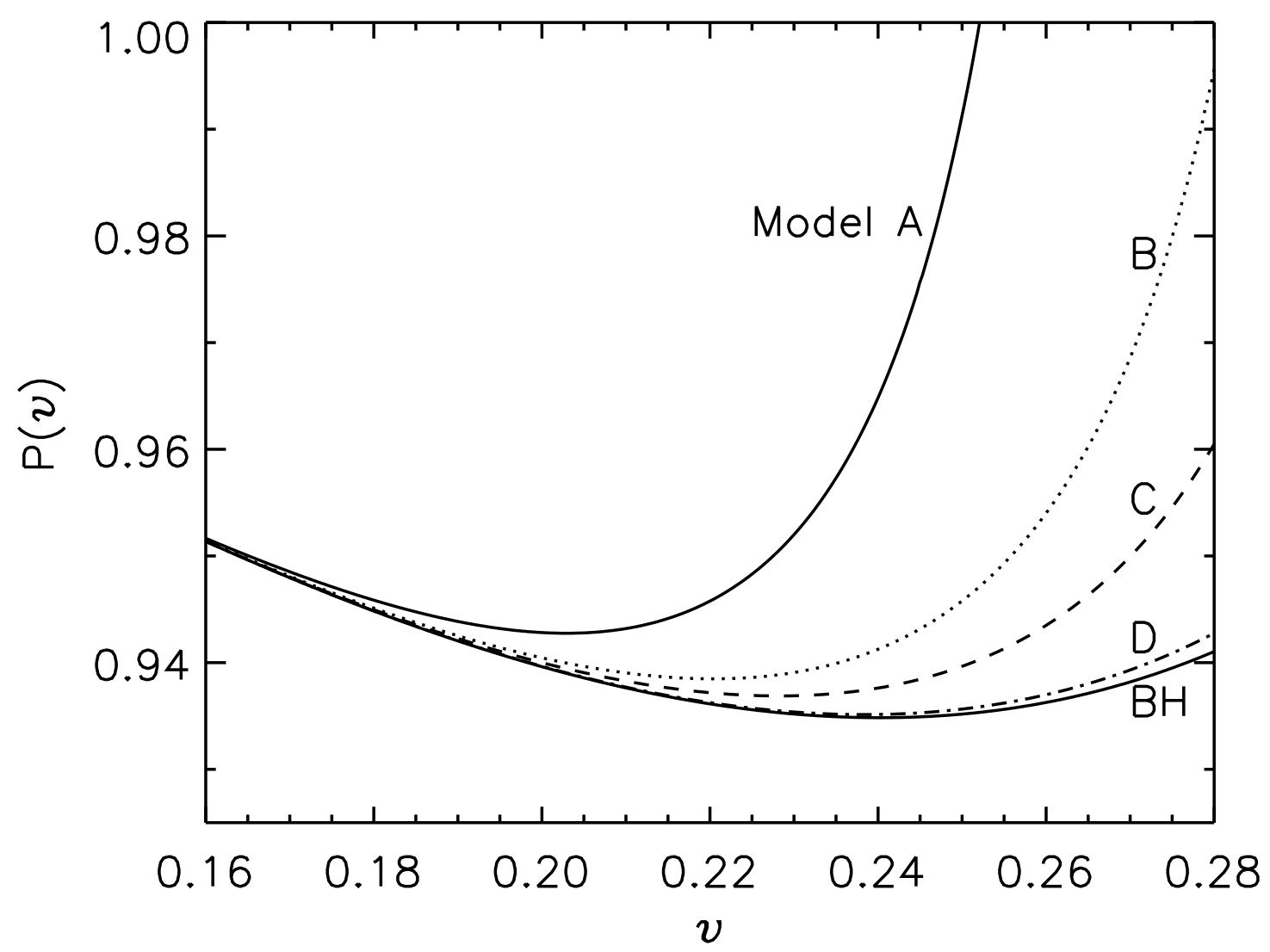

FIG. 2. The normalized energy flux, $P(v)$, is plotted as in Fig. 1, but for a smaller orbital velocity range, such that the peaks due to the excitation of the stellar modes are excluded. 


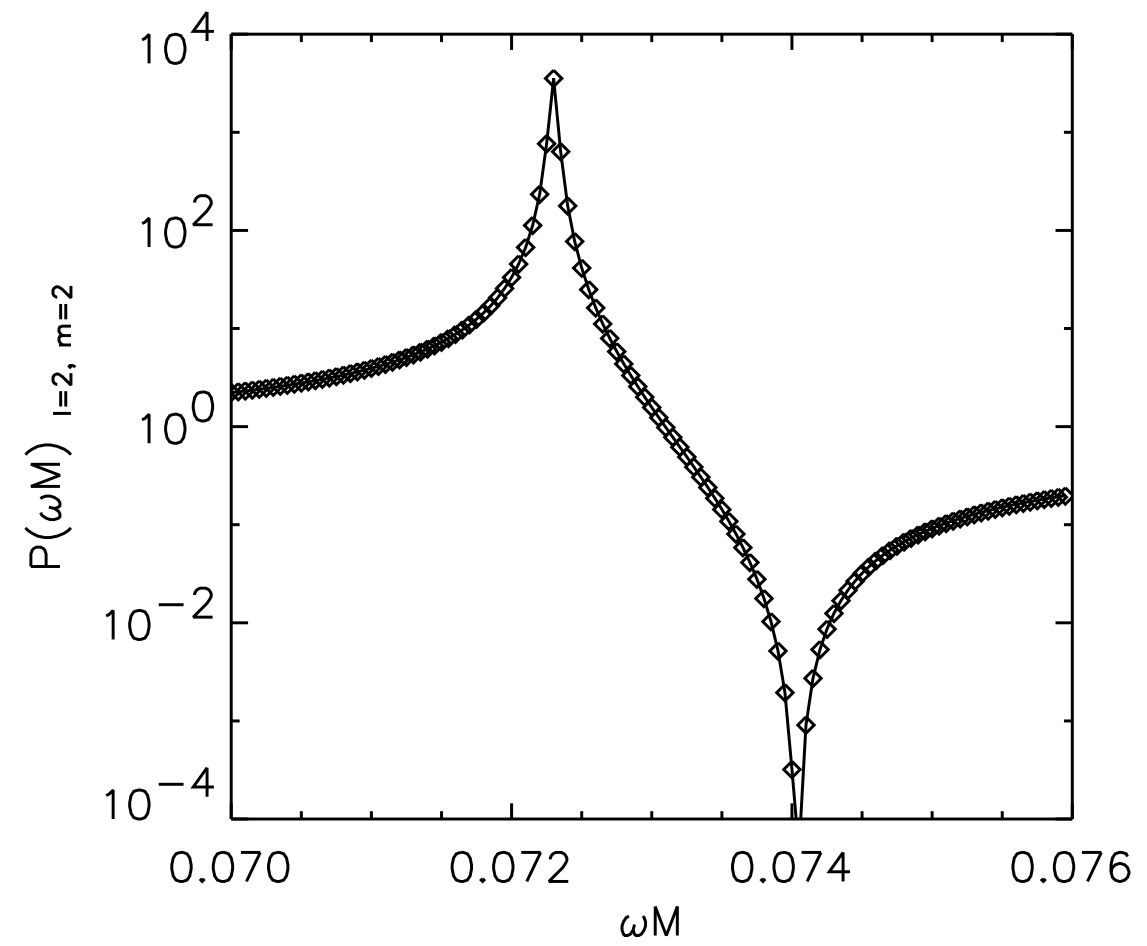

FIG. 3. The normalized energy flux emitted by the stellar model C, is computed by using the toy model (solid line) and by integrating the equations of stellar perturbations for $l=2$ and $m=2$ (diamonds), and plotted as a function of the dimensionless orbital frequency $\omega M$ near the resonance of the $f$-mode. The agreement is excellent. 


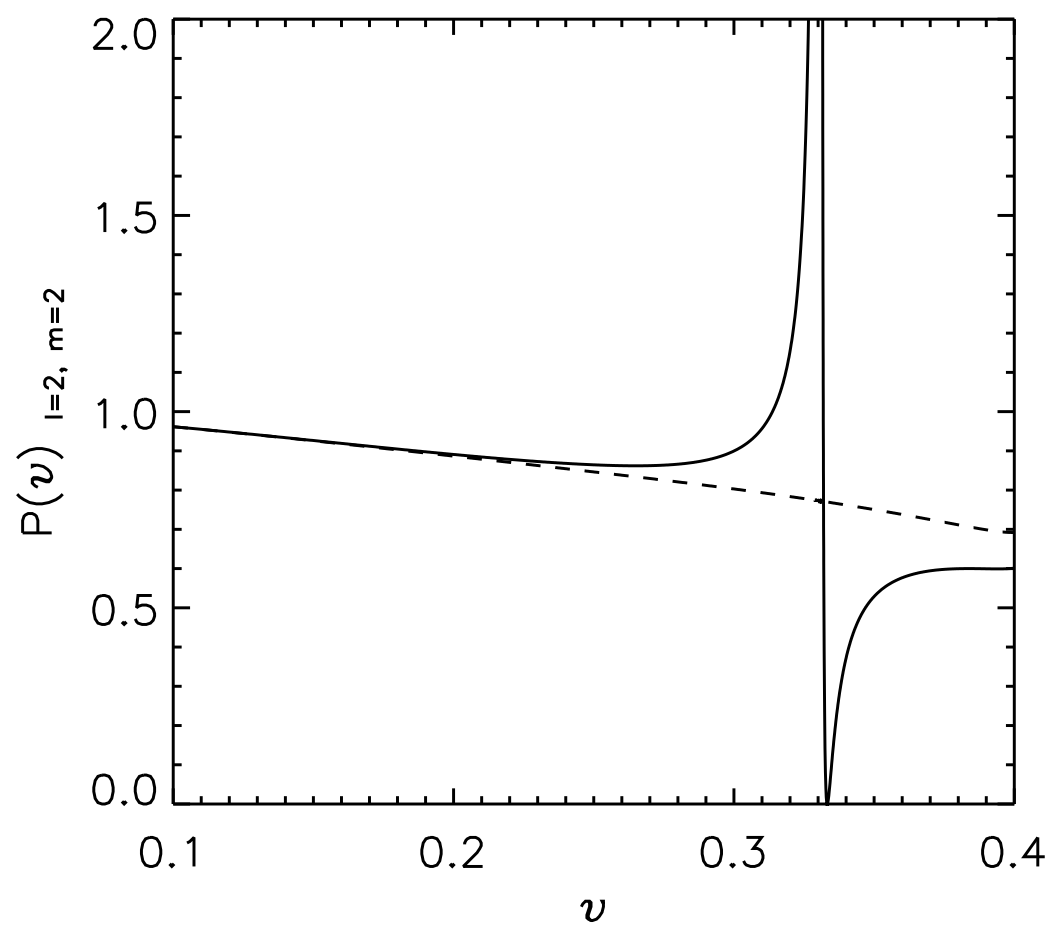

FIG. 4. The $l=m=2$ contribution to the normalized energy flux, $P_{l=2, m=2}(v)$, is plotted as a function of the orbital velocity (solid line), and compared to the result of subtracting the contribution of the resonance (dashes) using the analytical model, as described in the text. The data refer to the stellar model C. 


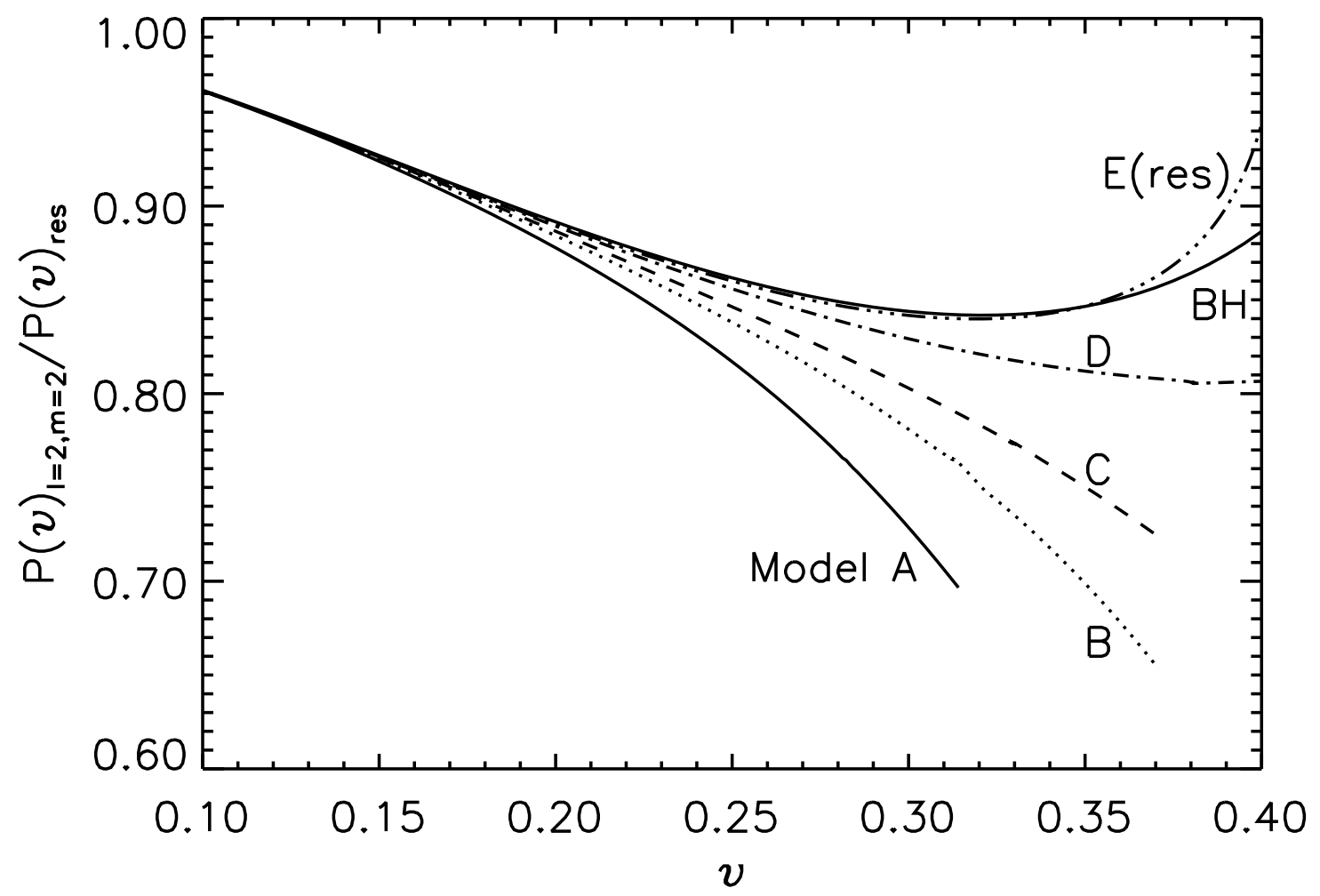

FIG. 5. In this figure we plot the normalized energy flux emitted by the four models of star for $l=2$ and $m=2$ versus the orbital velocity; the contribution of the resonances has been removed by dividing $P_{l=2, m=2}(v)$ by $P_{r e s}$, as described in Sec. III. For comparison, we plot the normalized energy flux emitted by the black hole $(B H)$ and by the stellar model $E(E($ res $))$; in this case we do not subtract the contribution of the resonant excitation of the quasi-normal modes because in order to excite these modes the point particle should move on an orbit of radius smaller than $6 \mathrm{M}$ (for the black hole), or smaller than the stellar radius (for model E). 

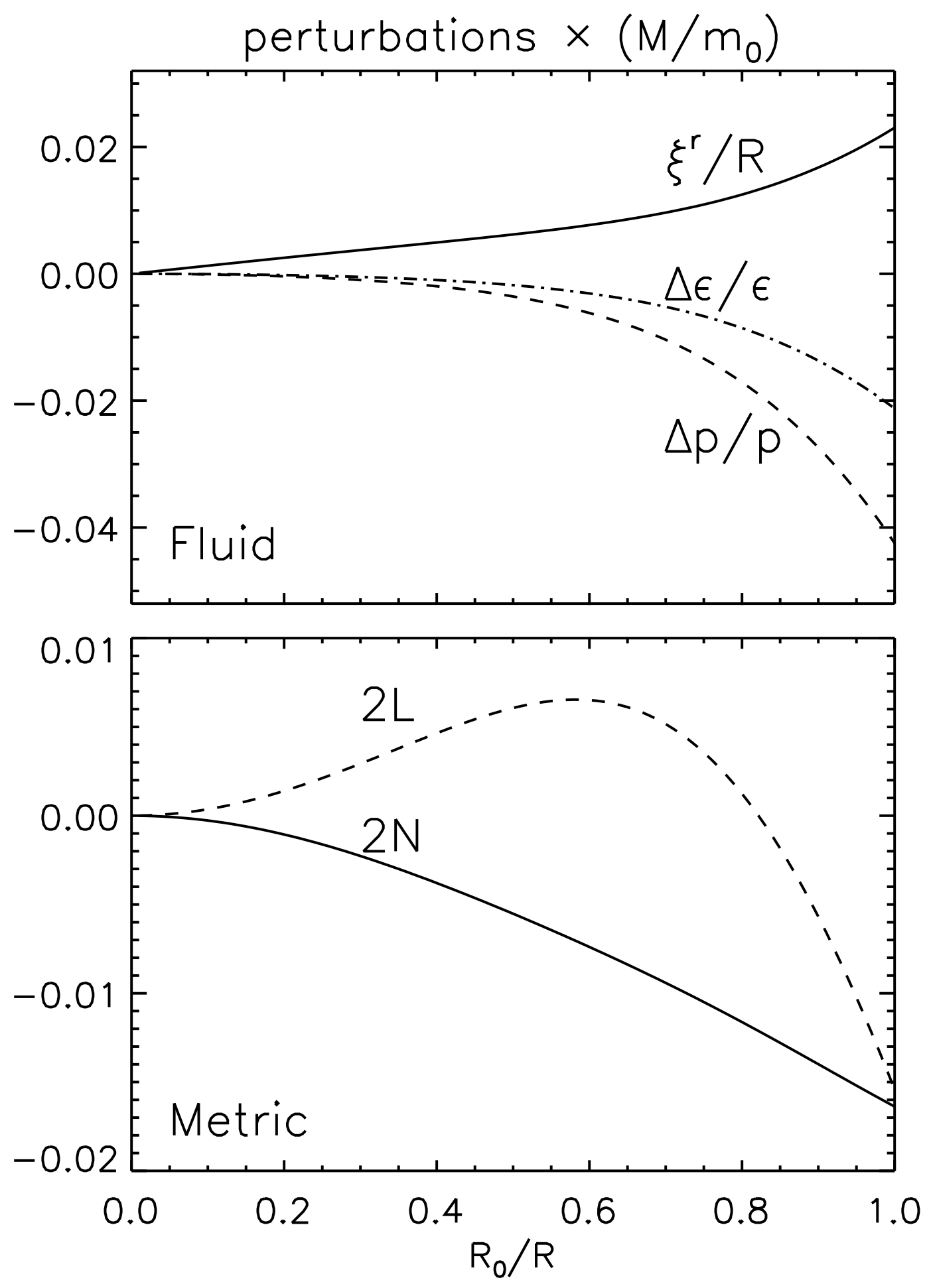
FIG. 6. This figure refers to the stellar model D perturbed by a point mass moving on a circular orbit of radius $R_{0}=3 R$. All quantities are normalized to the ratio $m_{0} / M$, and only the $l=2, m=2$ contribution is shown, which is the most significant. In the upper panel, the radial component of the lagrangian displacement normalized to the radius of the star, $\xi_{r}(r) / R$, and the lagrangian perturbations of the density and of the pressure normalized to their equilibrium values, $\Delta p(r) / p(r)$ and $\Delta \epsilon(r) / \epsilon(r)$, are plotted as a function of the radial distance from the center of the star. The metric perturbations $2 N$ and $2 L$ plotted in the lower panel, have to be compared to unity, since they are a measure of the deviations of the perturbed metric functions $\nu(r)$ and $\mu_{2}(r)$ with respect to their equilibrium values (cf. Paper I, Eq. (2.1)). 


\section{REFERENCES}

[1] L. Gualtieri, E. Berti, J.A. Pons, G. Miniutti, V. Ferrari, Phys Rev D 64, 104007, (2001).

[2] P.C. Peters, Phys. Rev. 136 n. 4b, 1224 (1964).

[3] S. Chandrasekhar, V. Ferrari, Proc. R. Soc. Lond. A432, 247 (1990).

[4] J.F. Zerilli, Phys. Rev D. 2, 2141 (1970).

[5] T. Regge, J.A. Wheeler, Phys. Rev. 108, 1063 (1957).

[6] J.M. Bardeen, W.H. Press, J. Math. Phys. 14, 7 (1973).

[7] S.A. Teukolsky, Ap. J. 185, 635 (1973).

[8] E. Poisson, Phys. Rev. D 52 n. 10, 5719 (1995).

[9] E. Poisson, Phys. Rev. D 55 n. 12, 7980 (1997).

[10] C. Cutler, L. S. Finn, E. Poisson, G. J. Sussman, Phys. Rev. D 47 n. 4, 1511 (1993).

[11] N. Andersson, K. D. Kokkotas, MNRAS 299, 1059 (1998).

[12] Y. Kojima, Prog. Theor. Phys. 77, 297, (1987).

[13] http://www.astro.cf.ac.uk/geo/euro/

[14] K. Taniguchi, E. Gourgoulhon, S. Bonazzola, Phys. Rev. D 64, 064012 (2001).

[15] T. Damour, B. R. Iyer, B. S. Sathyaprakash, Phys. Rev. D 57 n. 2, 885 (1998).

[16] W.C.G. Ho, D. Lai, MNRAS, 308, 153 (1999).

[17] L. Blanchet, to appear in Living Reviews in Relativity, gr-qc/0202016 (2002). 